
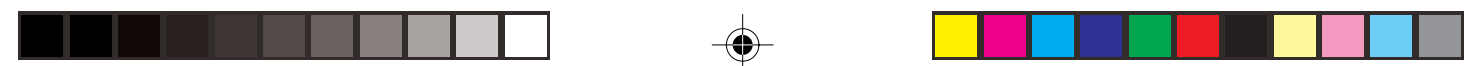

\title{
LOS MAMÍFEROS DE LA RESERVA ECOLÓGICA HUITEPEC, CHIAPAS, MEXICO
}

\author{
EDUARDO J. NARANJO ${ }^{1}$ Y EDUARDO ESPINOZA MEDINILLA $^{2}$
}

${ }^{1}$ El Colegio de la Frontera Sur, Apartado postal 63, San Cristóbal de las Casas, Chiapas 29290 México.enaranjo@sclc.ecosur.mx ${ }^{2}$ Instituto de Ecología Universidad Nacional Autónoma de México Ap. Postal 70-275, 04510 México D.F.

Resumen. En este trabajo presentamos un listado de mamíferos silvestres de la Reserva Ecológica Huitepec (136 ha), ubicada en el municipio de San Cristóbal de Las Casas, en los altos de Chiapas, México. Entre 1991 y 1998 encontramos 40 especies de mamíferos pertenecientes a 15 familias y 7 órdenes. Los órdenes con mayor número de especies fueron Rodentia ( $\mathrm{n}=17$ especies) y Chiroptera ( $\mathrm{n}=11$ especies). Dos de las especies presentes en el área de estudio (Sorex stizodon y Peromyscus zarhynchus) son endémicas de México y Guatemala, éstas dos, más otras cinco especies se encuentran en algún estatus de riesgo, por lo que se incluyen en la NOM-ECOL-059-2000. Considerando la pequeña superficie que ocupa la Reserva Huitepec y el número relativamente alto de especies de mamíferos con presencia verificada en la misma, se infiere que esta área protegida constituye una muestra importante de la diversidad mastofaunística de la meseta central de Chiapas. Sin embargo, el acelerado incremento de la población humana local, la expansión territorial de la ciudad de San Cristóbal, y las condiciones de marginación y pobreza de la población rural de la región constituyen serios factores de riesgo para la permanencia de la REH y su biodiversidad en el futuro inmediato.

\begin{abstract}
We present a checklist of the mammals found in Huitepec Ecological Reserve, located in the municipality of San Cristóbal de Las Casas, Chiapas, México. Between 1991 and 1998 we found 40 mammal species of 15 families and 7 orders. The most specious mammalian orders in the study area were Rodentia ( $\mathrm{n}=17$ species), and Chiroptera ( $\mathrm{n}=11$ species). Two of the species (Sorex stizodon and Peromyscus zarhynchus) present in the Huitepec Reserve are endemic of México and Guatemala. These and other five species are enlisted in the Mexican Norm of Endangered Wildlife (NOM-ECOL-059-2000). Considering the small size of the Huitepec Reserve as well as its relatively high number of mammal species, it is possible to infer that this protected area constitutes an important sample of the mammalian diversity in the Chiapas highlands. However, the high growth rate of the local human population, the expansion of the city of San Cristóbal, and the conditions of poverty and abandonment suffered by the rural population in the region constitute serious threats to the persistence of the Huitepec Reserve and its biodiversity in the near future.
\end{abstract}

Palabras clave: Bosque mesófilo, Chiapas, mamíferos, Reserva Huitepec, San Cristóbal de Las Casas. Key words: Chiapas, cloud forest, Huitepec Reserve, mammals, Mexico, San Cristobal de Las Casas. 


\section{INTRODUCCIÓN}

México es reconocido entre los países con mayor diversidad de mamíferos en el mundo (Arita y Ceballos 1997; Cervantes et al., 1994; Ramírez-Pulido et al., 1996). En el país se tienen listados prácticamente completos tanto a nivel de grandes regiones geográficas como por estados de la república. El estado de Chiapas ocupa el segundo lugar nacional en cuanto a riqueza de especies de mamíferos (Retana y Lorenzo, 2002), además de ser una de las entidades con mayor número de estudios mastozoológicos (Guevara et al., 2001). La mayor parte de estos estudios han consistido en inventarios de especies, descripciones taxonómicas y análisis de distribución principalmente de roedores y quirópteros en algunas localidades (Alvarez y Alvarez 1991; Espinoza et al., 1998; Medellín 1994; Medellín et al., 1986). Sin embargo, aún estamos lejos de conocer completamente la mastofauna de la mayoría de las numerosas áreas silvestres protegidas y no protegidas de Chiapas (Flores y Gerez 1994).

Los inventarios de especies representan información útil para la toma de decisiones sobre acciones conservación y manejo de la vida silvestre en las áreas naturales protegidas (Cervantes et al., 1994; Wilson y Reeder 1993). Por ello, es importante conocer las especies que se distribuyen dentro de los límites de dichas áreas y reconocer su potencial como reservas de biodiversidad. La Reserva Ecológica Huitepec (REH) fue creada en 1987 como la primera reserva biológica privada en México, y es la única que conserva bosque mesófilo de montaña en la región de los altos de Chiapas. El presente trabajo tuvo como objetivo central obtener un listado actualizado de las especies de mamíferos que habitan los bosques de la REH.

\section{ÁREA DE ESTUDIO}

La Reserva Ecológica Huitepec (REH; 1644'38' N y 92 40’15' O), fue creada en 1987 como la primera reserva privada de México y es administrada por PRONATURA, A.C. El área protegida incluye 136 ha ubicadas en las laderas E y NE del Cerro Huitepec a $6 \mathrm{~km}$ del extremo oeste de la ciudad de San Cristóbal de Las Casas, Chiapas (Anónimo 1987; Ramírez et al., 1998; Figura 1). La reserva consta de una serie de laderas con pendientes pronunciadas (40-60\%), con alturas que van desde los 2,230 hasta los 2,710 m.s.n.m. Esta área constituye uno de los últimos remanentes de bosque primario en la porción central de los altos de Chiapas.

La vegetación característica de la REH consiste en bosque de encino y bosque mesófilo de montaña destacando la casi total ausencia de coníferas con excepción de algunas comunidades de pino-encino en las partes intermedias y bajas de la ladera oeste, las cuales quedan fuera de los limites de la reserva. Dentro del área protegida se 


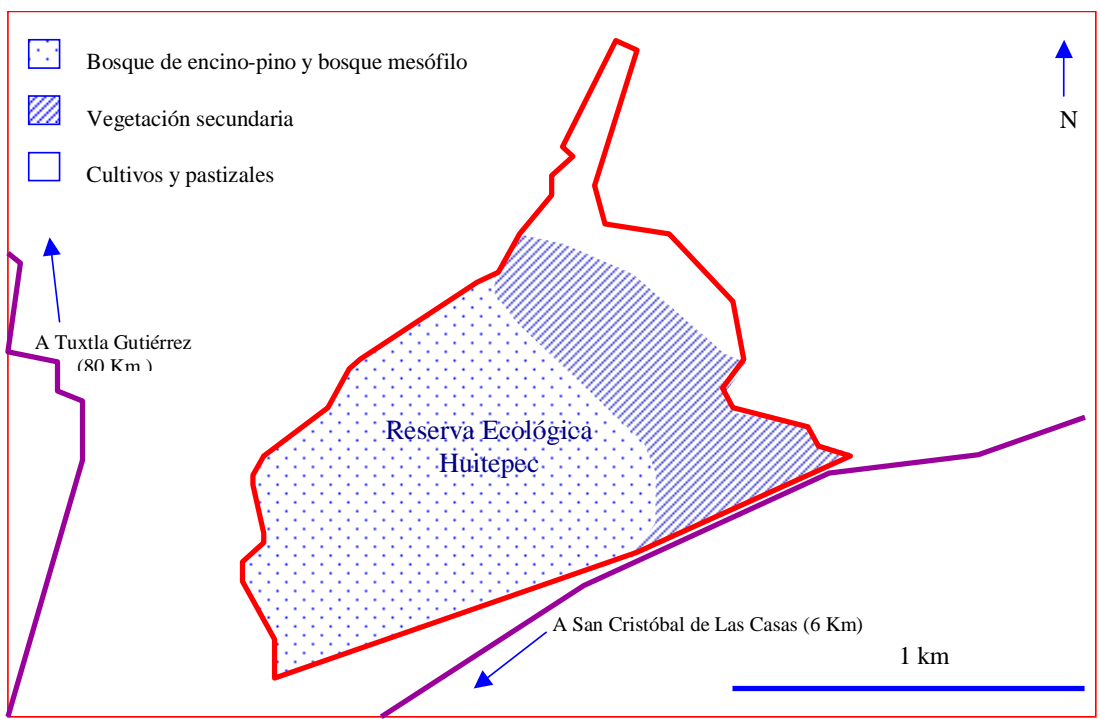

Figura 1. Área y tipos de vegetación de la Reserva Ecológica del Huitepec. También se señalan las distancias hacia las principales ciudades.

han registrado 315 especies de plantas vasculares, lo que representa el 32\% de la riqueza florística estimada para la región ubicada por encima de los 2,000 metros de altura en la meseta central de Chiapas (Ramirez et al., 1998).

\section{MÉTODOS}

Por el pequeño tamaño del área de estudio, se utilizaron como transectos los andadores turísticos y de vigilancia, pues estos abarcan los tres tipos básicos de vegetación (pastizal, bosque encino-pino y bosque mesófilo de montaña). Entre 1991 y 1998, ejemplares de especies terrestres pequeñas de mamíferos (roedores, insectívoros, marsupiales y pequeños carnívoros) fueron capturados con trampas Sherman (cebadas con avena y esencia de vainilla), y Tomahawk (cebadas con plátano y sardina), dispuestas en series de dos transectos por tipo de vegetación, de manera que cubrieran las áreas más representativas de la REH. Los quirópteros fueron capturados con redes de niebla colocadas aleatoriamente en los transectos. Solamente se colectaron de 3 a 5 ejemplares por especie y tipo de vegetación. Los individuos colectados se identificaron mediante las claves especializadas de Alvarez et al., (1994), Hall (1981), Medellín et al., (1997) y Reid (1997). De los ejemplares se obtuvieron las medidas convencionales, edad, sexo y estado reproductivo y se prepararon para su 
ingreso a las colecciones mastozoológicas de El Colegio de la Frontera Sur (ECO-SCM ) y del Instituto de Historia Natural y Ecología de Chiapas (IHNMASTO). Las especies medianas y grandes (edentados, carnívoros, lagomorfos y artiodáctilos) fueron detectadas mediante la observación de individuos y/o sus rastros (huellas) en recorridos diurnos y nocturnos de los transectos, apoyándose en las guías de campo de Aranda (1981) y Aranda y March (1987). De estos órdenes no se realizaron colectas de ejemplares, sino únicamente registros fotográficos y moldes de yeso para las huellas.

\section{RESULTADOS Y DISCUSIÓN}

En la REH se encuentran 7 órdenes, 15 familias y 40 especies de mamíferos (Anexo 1). Los órdenes con mayor número de especies son los roedores, con 17 (42.5\% del total) y los quirópteros, con 11 (29.7\%). Además de las enlistadas en el Anexo 1, es muy probable que en el área existan cuando menos 12 especies más de mamíferos (Anexo 2), que han sido capturadas tanto en las inmediaciones del volcán Huitepec, como en otras localidades cercanas dentro del municipio de San Cristóbal de Las Casas (i.e., San José Bocomtenelté, 4 km al sur de la REH). Alba et al., (1995) registraron 45 especies de mamíferos para el municipio de San Cristóbal de Las Casas (484 km²), en tanto que Retana y Lorenzo (2002) presentaron una lista de 204 especies presentes en todo el estado de Chiapas $\left(75,634 \mathrm{~km}^{2}\right.$; Figura 2). Considerando la extensión tan limitada $\left(0.136 \mathrm{~km}^{2}\right)$ y el número de especies de mamíferos con presencia verificada en la REH $(n=40)$, se infiere que esta área protegida constituye una muestra importante de la diversidad mastofaunística de la meseta central de Chiapas.

En cuanto a su distribución, 13 especies presentes en la REH son compartidas tanto con Norteamérica como con Sudamérica, siete especies son compartidas sólo Norteamérica, y siete más solamente con Sudamérica (Figura 3). Nueve de las especies de la REH son endémicas de Mesoamérica, y dos de ellas endémicas de México y Guatemala (Sorex stizodon y Peromyscus zarhynchus).

De las especies de mamíferos presentes en la REH, siete se encuentran en algún estatus de riesgo de acuerdo a la SEMARNAP (NOM-059-ECOL-2000): cuatro especies están sujetas a protección especial (Cryptotis goldmani, C. parva, Sorex stizodon y Peromyscus zarhynchus), y tres se consideran amenazadas (Sphiggurus mexicanus, Glaucomys volans y Peromyscus boylii).

La Reserva Ecológica Huitepec significa un refugio importante para poblaciones de la mayoría de las especies de mamíferos silvestres del municipio de San Cristóbal de Las Casas. Sin embargo, el acelerado incremento de la población humana, la consiguiente expansión territorial de la ciudad de San Cristóbal, y las condiciones de marginación 

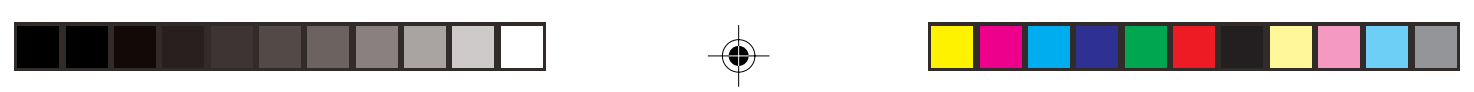

vol. 5

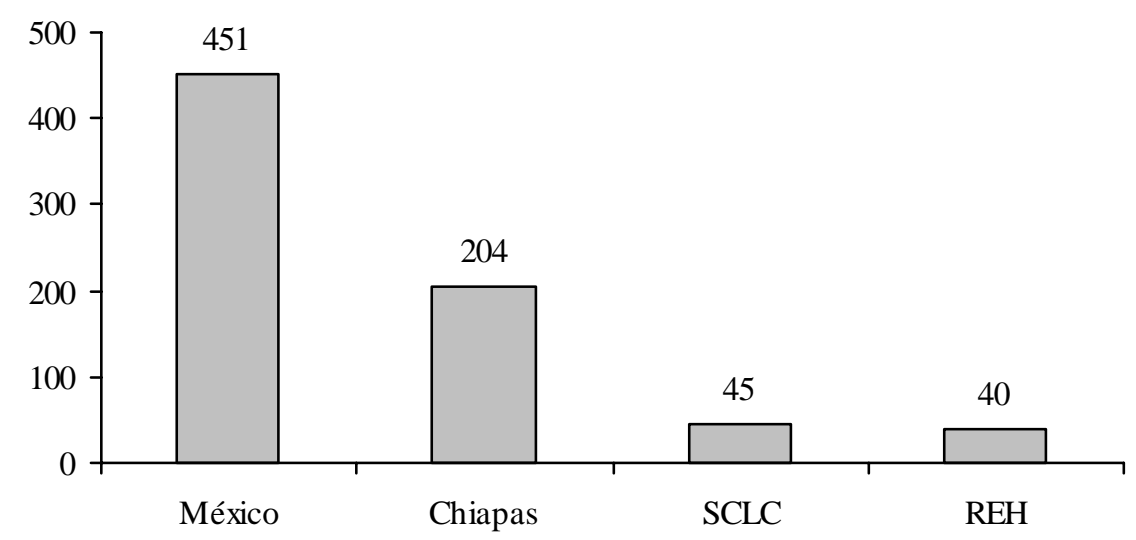

Figura 2. Números comparativos de especies de mamíferos presentes en México, Chiapas, San Cristóbal de Las Casas (SCLC) y la Reserva Ecológica Huitepec (REH).

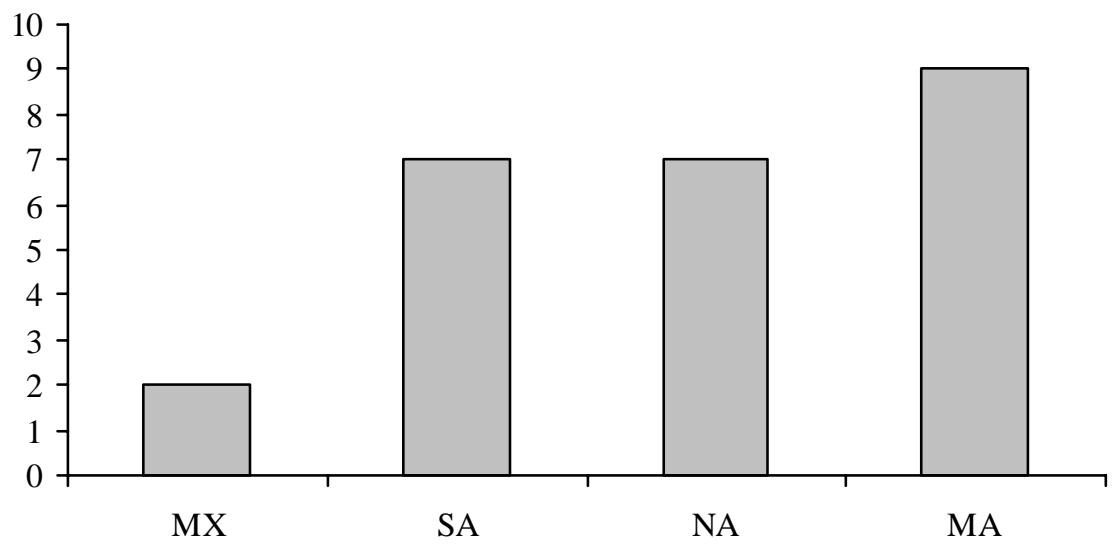

Figura 3. Número de especies de mamíferos de acuerdo a su rango de distribución (MX= endémicas de México; SA= compartidas con Sudamérica; NA= compartidas con Norteamérica; MA=endémicas de Mesoamérica; $\mathrm{AM}=$ compartidas con Norteamérica y Sudamérica. 
y pobreza de la población rural de la región de los altos de Chiapas, sin duda representan y representarán serios factores de riesgo para la permanencia de la REH y su biodiversidad en el futuro inmediato.

\section{AGRADECIMIENTOS}

Agradecemos las facilidades otorgadas por PRONATURA, A.C., la Universidad de Ciencias y Artes de Chiapas, el Instituto de Historia Natural de Chiapas y El Colegio de la Frontera Sur para la realización de este trabajo. Agradecemos también la colaboración de Carlos Marina, Guillermo Bond, Darío Navarrete, Jorge Bolaños, Luis Alfredo Jiménez y David López en el trabajo de campo.

\section{LITERATURA CITADA}

Alba, M. P., D. A. Navarrete e I. Fuentes. 1995. Fauna del Municipio de San Cristóbal de las Casas. Serie de divulgación Fauna de México, Cuaderno 1. El Colegio de la Frontera Sur. San Cristóbal de Las Casas, Chiapas, México.

Alvarez, T., S. T. Alvarez y J. C. López-Vidal. 1994. Claves para murciélagos mexicanos. Centro de Investigaciones Biológicas del Noroeste e Instituto Politécnico Nacional, Escuela Nacional de Ciencias Biológicas. La Paz, Baja California Sur, México.

Alvarez, S. y T. Alvarez. 1991. Los murciélagos de Chiapas. Escuela Nacional de Ciencias Biológicas, Instituto Politécnico Nacional, México, D. F.

Anónimo 1987. Huitepec, primera estación biológica de Pronatura. Pronatura A. C., San Cristóbal de Las Casas, Chiapas. Inédito. 8 pp.

Aranda, J. M. 1981. Rastros de los mamíferos silvestres de México. Manual de campo. Instituto Nacional de Investigaciones sobre Recursos Bióticos, Xalapa, Veracruz, México.

Aranda, J. M. e I. J. March. 1987. Guía de los mamíferos silvestres de Chiapas. Instituto Nacional de Investigaciones sobre Recursos Bióticos. México, D. F.

Arita, H. T. y G. Ceballos. 1997. Los mamíferos de México: Distribución y estado de conservación. Revista Mexicana de Mastozoología 2:33-71.

Cervantes, F. A., A. Castro-Campillo y J. Ramírez-Pulido. 1994. Mamíferos terrestres nativos de México. Anales del Instituto de Biología, 65 (1) 176-190.

Espinoza, E. E., A. Anzures y E. Cruz. 1998. Mamíferos de la Reserva de la Biósfera El Triunfo, Chiapas. Revista Mexicana de Mastozoología 3:79-94.

Flores-Villela, O. y O. Gerez. 1994. Biodiversidad y conservación en México: vertebrados, vegetación y uso del suelo. Comisión Nacional para el Conocimiento y Uso de la Biodiversidad, México, D. F.

Guevara-Chumacero, L. M., R. López-Wilchis \& V. Sánchez-Cordero. 2001. 105 años de investigación mastozoológica en México (1890-1995): una revisión de sus enfoques y tendencias. Acta Zoológica Mexicana. 83:48-63.

Hall, E. R. 1981. The mammals of North America. John Wiley, New York, USA. 
Medellín, R. A. 1994. Mammal diversity and conservation in the Selva Lacandona, Chiapas, México. Conservation Biology 8:780-799.

Medellín, R. A., H. T. Arita y O. Sánchez. 1997. Identificación de los murciélagos de México, clave de campo. Asociación Mexicana de Mastozoología, A. C. México, D. F.

Medellín, R. A., G. Urbano, O. Sánchez, G. Téllez y H. Arita. 1986. Notas sobre los murciélagos del Este de Chiapas. The Southwestern Naturalist 3:532-535.

Ramírez, N., S. Ochoa, M. González y P. Quintana. 1998. Análisis florístico y sucesional en la Estación Biológica Cerro Huitepec, Chiapas, México. Acta Botánica Mexicana 44:59-85.

Ramírez-Pulido, J., A. Castro, J. Arroyo y F. Cervantes. 1996. Lista taxonómica de los mamíferos de México. Occas. Papers Mus. Texas Tech University, 158:1-62.

Reid, F. A. 1997. A field guide to the mammals of Central America and Southeast Mexico. Oxford University Press, New York.

Retana, O. G. y C. Lorenzo. 2001. Lista de los mamíferos terrestres de Chiapas: endemismos y estado de conservación. Acta Zoológica Mexicana 85:25-49.

Secretaría de Medio Ambiente, Recursos Naturales y Pesca (SEMARNAP). 2000. Norma Oficial Mexicana NOM-059-ECOL-2000. Protección ambiental, especies de flora y fauna silvestres de México, categorías de riesgo y especificaciones para su inclusión, exclusión o cambio, y lista de especies en riesgo. Diario Oficial de la Federación 1:1-62.

Wilson, D. E. y D. M. Reeder. 1993. Mammal species of the world. Segunda Edición. Smithsonian Institution Press, Washington, D. C. 

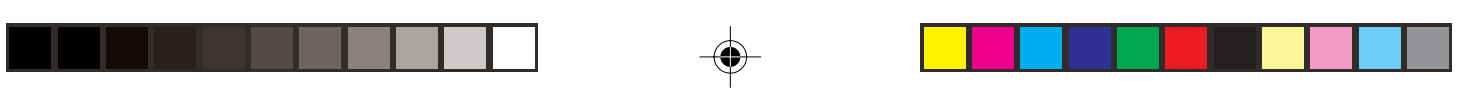

Diciembre 2001

Naranjo P. y E. Espinoza - Mamíferos de Huitepec, Chiapas

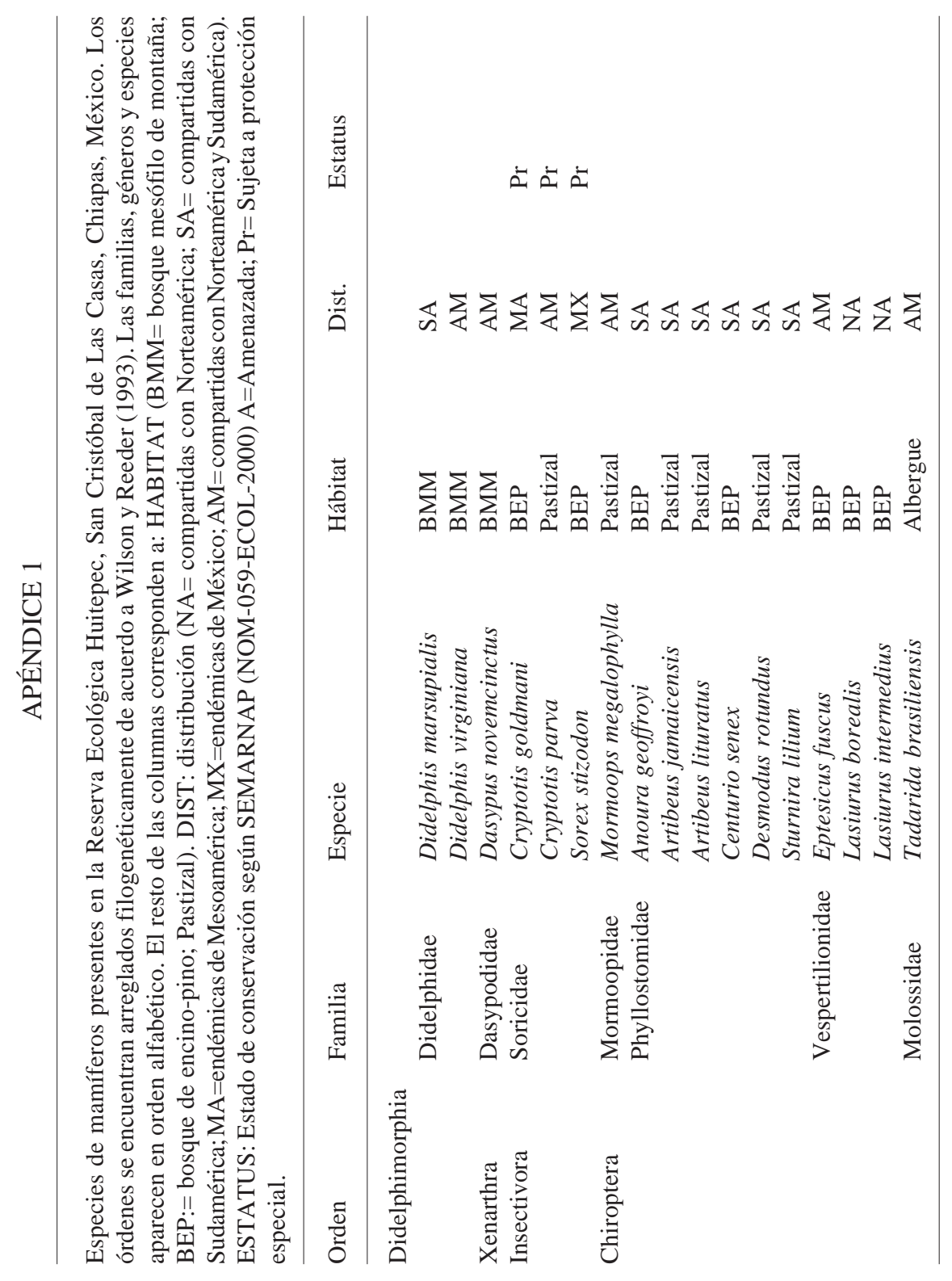




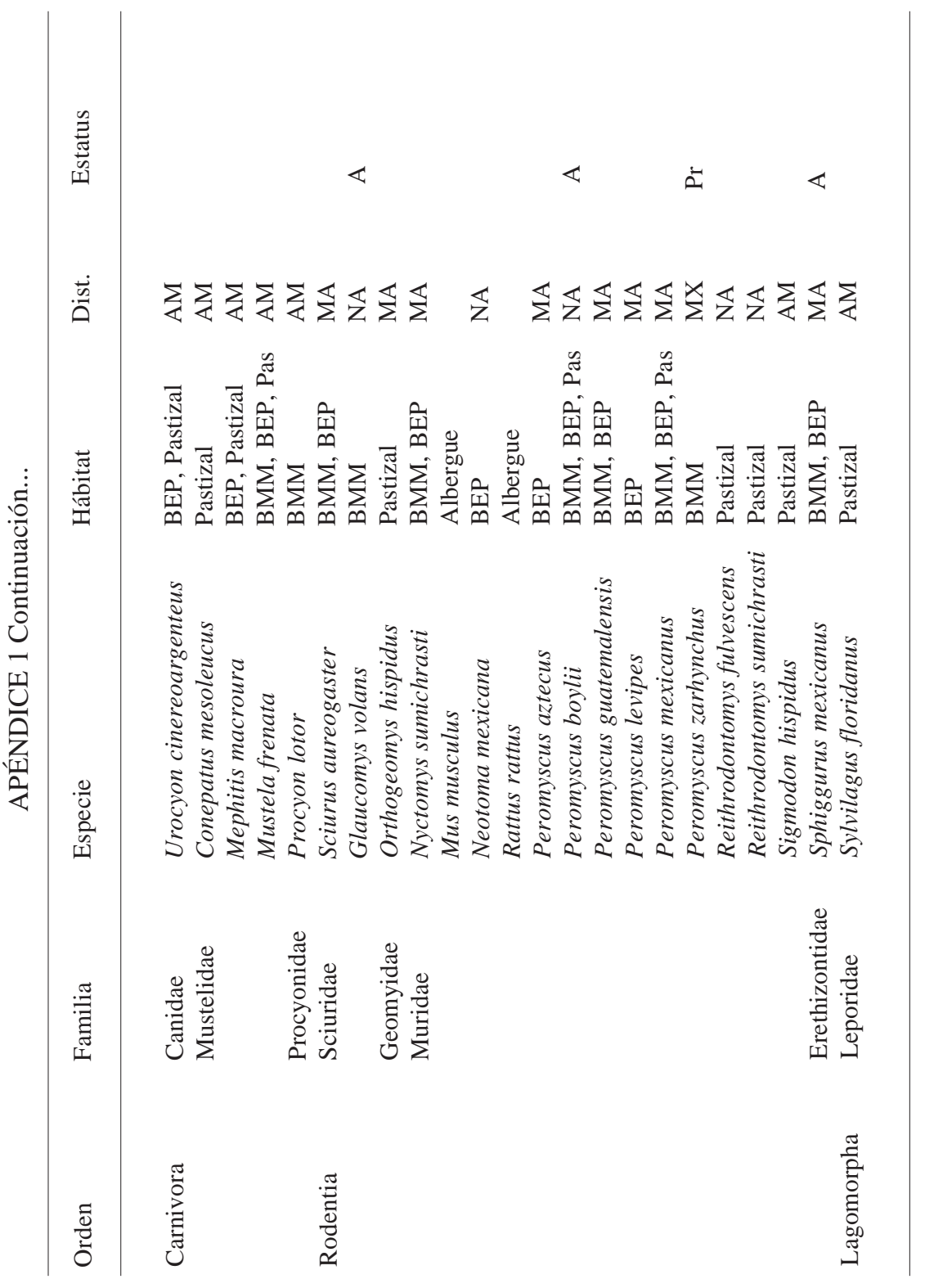



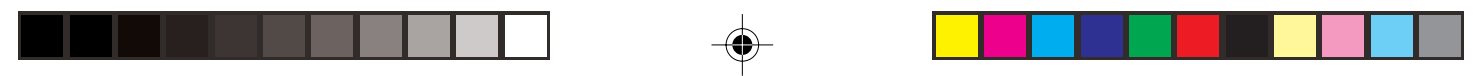

Diciembre 2001

\section{APÉNDICE 2}

Especies de mamíferos potencialmente presentes en la Reserva Ecológica Huitepec, San Cristóbal de Las Casas, Chiapas, México.

\begin{tabular}{lll}
\hline ORDEN & FAMILIA & ESPECIE \\
\hline Insectivora & Soricidae & $\begin{array}{l}\text { Sorex saussurei } \\
\text { Sorex veraepacis }\end{array}$ \\
Chiroptera & Phyllostomidae & Sturnira ludovici \\
& Vespertilionidae & Myotis keaysi \\
& Myotis nigricans \\
& Myotis thysanodes \\
& Myotis velifer \\
Rodentia & Maiomys musculus \\
& & Habromys lophurus \\
& & Oryzomys alfaroi \\
& Reithrodontomys megalotis \\
\end{tabular}

